\title{
Insulin resistance, blood glucose and inflammatory cytokine levels are risk factors for cardiovascular events in diabetic patients complicated with coronary heart disease
}

\author{
CHANGMEI WANG, FANG LI, JINGJING GUO, CONGCONG LI, DASHUAI XU and BIN WANG \\ Department of Geriatrics, Jinan Central Hospital, Shandong University, \\ Jinan, Shandong 250013, P.R. China
}

Received June 28, 2017; Accepted November 20, 2017

DOI: $10.3892 /$ etm.2017.5584

\begin{abstract}
This study was designed to investigate the relation of insulin resistance, blood glucose and inflammatory cytokines with cardiovascular events in diabetic patients complicated with coronary heart disease (CHD). A total of 120 patients with diabetes mellitus type 2 (T2DM) complicated with CHD admitted to Jinan Central Hospital from January 2015 to March 2016 were enrolled in this study. There were 60 cases complicated with cardiovascular events and 60 had no history of cardiovascular events; there were 40 cases with abnormal blood glucose, 40 with insulin resistance and 40 with elevated inflammatory cytokines. Changes in the levels of blood glucose, fasting serum insulin and inflammatory cytokines as well as changes in the homeostasis model assessment of insulin resistance indexes (HOMA-IR) were recorded and compared among groups of patients. Besides, changes in the Global Registry of Acute Coronary Events (GRACE) risk score and the incidence rate of cardiovascular events were also detected and multivariate logistic regression analysis was conducted so as to identify relevant risk factors. Our results showed the fasting blood glucose and the $2 \mathrm{~h}$ postprandial blood glucose levels in the non-cardiovascular event group were lower than those in the cardiovascular event group $(\mathrm{P}<0.05)$. However, levels of fasting serum insulin and HOMA-IR in the noncardiovascular event group were significantly higher than those in the cardiovascular event group $(\mathrm{P}<0.05)$. Additionally, levels of tumor necrosis factor- $\alpha$ (TNF- $\alpha)$, interleukin- 6 and C-reactive protein in the non-cardiovascular event group were significantly lower than those in the cardiovascular event group $(\mathrm{P}<0.05)$. Moreover, GRACE risk scores in patients with elevated inflammatory cytokines were higher than those in
\end{abstract}

Correspondence to: Dr Bin Wang, Department of Geriatrics, Jinan Central Hospital, Shandong University, 105 Jiefang Road, Lixia, Jinan, Shandong 250013, P.R. China

E-mail: wangbinjnzxyy@126.com

Key words: coronary heart disease, diabetes, blood glucose, insulin resistance, inflammatory factors, cardiovascular events patients with insulin resistance and those in patients with blood glucose abnormalities $(\mathrm{P}<0.05)$. The incidence rate of cardiovascular events in patients with blood glucose abnormalities was lower than that in patients with insulin resistance and that in patients with elevated inflammatory cytokines. There was a positive correlation between TNF- $\alpha$ and HOMA-IR $(\mathrm{P}<0.05)$, and between HOMA-IR and the GRACE risk score $(\mathrm{P}<0.05)$. Blood glucose abnormalities, insulin resistance and inflammatory cytokines were all independent risk factors for cardiovascular events. Based on our findings, stronger inflammatory responses in patients with T2DM complicated with CHD lead to higher incidence rates of cardiovascular events. Besides that, elevated blood glucose and insulin resistance levels are also independent risk factors for cardiovascular events.

\section{Introduction}

Elevated blood glucose, insulin resistance and inflammation marker lnt of simple coronary heart disease (CHD) or diabetes mellitus type 2 (T2DM) (1), which, in turn, also affect the body's fat metabolism, blood pressure regulation and glucose metabolism process (2). T2DM and CHD are associated with decreased sensitivity to insulin, weakened blood pressure regulation ability (3), damaged vascular endothelial cells, dysfunction of the fibrinolytic system (4), and enhancement of the inflammatory responses (5). However, the way in which these factors interact with each other to result in either or both diseases is not clear. In order to better explore the effects of changes in blood glucose, insulin resistance and inflammatory factors on cardiovascular events in patients with T2DM complicated with CHD, this study obtained data from a group of patients and analyzed it statistically; the results are presented below.

\section{Patients and methods}

General data. A total of 120 patients with T2DM complicated with CHD admitted to Jinan Central Hospital from January of 2015 to March of 2016 were selected. All patients were diagnosed with CHD by coronary angiography and with T2DM by a glucose tolerance test. The Ethics Committee of the hospital 
Table I. Comparison of changes in blood glucose between patients with cardiovascular events and those without cardiovascular events (mmol/l, mean $\pm \mathrm{SD})$.

\begin{tabular}{lcc}
\hline Groups & $\begin{array}{c}\text { Fasting } \\
\text { blood }\end{array}$ & $\begin{array}{c}\text { 2 h postprandial } \\
\text { blood glucose }\end{array}$ \\
\hline Non-cardiovascular & $5.3 \pm 1.1$ & $7.0 \pm 1.6$ \\
Cardiovascular & $8.8 \pm 1.4$ & $14.4 \pm 2.4$ \\
t-test & 12.433 & 17.327 \\
P-value & $<0.05$ & $<0.05$ \\
\hline
\end{tabular}

SD, standard deviation.

approved the study and all participating patients signed informed consent forms. Patients complicated with malignant tumors, mental illnesses and immune system diseases and those taking glucocorticoids and/or immunosuppressive agents for a long time were excluded.

There were 60 cases with cardiovascular events, including 33 males and 27 females, aged $50-75$ years $(67.9 \pm 1.1$ on average). The patients in that group had lived with diabetes mellitus for 5 to 35 years $(20.1 \pm 3.1$ on avaerage) and with CHD for 5-30 years (15.2 \pm 1.1 on average). There were 60 patients in the group with no cardiovascular events, including 34 males and 26 females, aged 50-75 years $(67.8 \pm 1.2$ on average). These patients had suffered from diabetes mellitus for 5-35 years (20.0 \pm 3.0 on average) and CHD for 5-30 years $(15.3 \pm 1.0$ on average).

Methods. Changes in the levels of blood glucose, fasting serum insulin (FINS) and inflammatory cytokines as well as in the homeostasis model assessment of insulin resistance indexes (HOMA-IR) in patients with or without cardiovascular events were statistically analyzed. Besides, changes in Global Registry of Acute Coronary Events (GRACE) risk score and the incidence rate of cardiovascular events were detected, and the relationship between tumor necrosis factor- $\alpha$ (TNF- $\alpha$ ) and HOMA-IR and between HOMA-IR and GRACE risk score were analyzed. Multivariate logistic regression analysis was conducted to evaluate the correlation of elevated blood glucose, insulin resistance and inflammatory cytokine levels with cardiovascular events in diabetic patients complicated with CHD.

Observation indexes and evaluation methods. Cardiovascular events included heart failure, myocardial infarction, recurrent myocardial infarction, angina pectoris, recurrent angina and death due to any of these diseases. The fasting blood glucose and $2 \mathrm{~h}$ postprandial blood glucose levels were both detected loading venous blood samples into a Hitachi 7080 automatic biochemical analyzer. The normal value of fasting blood glucose was assumed at 3.9-6.1 mmol/1, while that of $2 \mathrm{~h}$ postprandial blood glucose was $7.8 \mathrm{mmol} / \mathrm{l}$. TNF- $\alpha$ and interleukin-6 (IL-6) were detected by using the double-antibody single-step sandwich method, and C-reactive protein (CRP) was detected by using the turbidimetric immunoassay. The results were based on

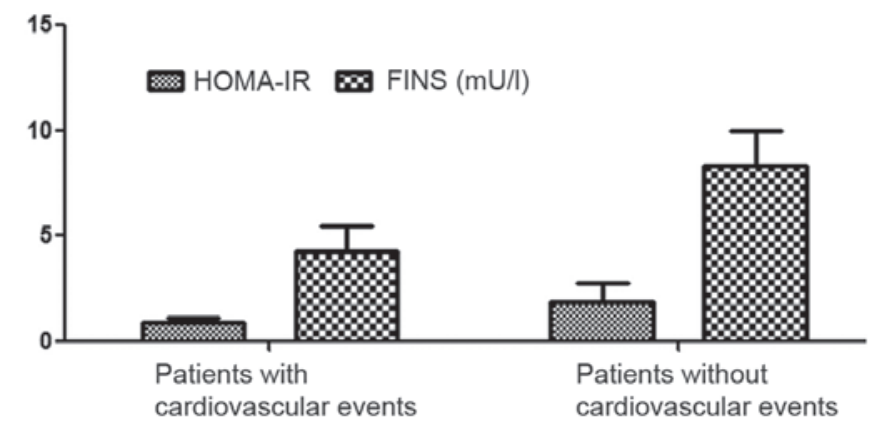

Figure 1. Comparisons of FINS and HOMA-IR between patients with cardiovascular events and those without cardiovascular events. FINS, fasting serum insulin; HOMA-IR, homeostasis model assessment of insulin resistance indexes.

the normal reference range of adult' TNF- $\alpha$ at $5-100 \mathrm{ng} / 1$, and that of adult IL-6 at 56.4-150.3 ng/l. The normal reference range for adult CRP was $\leq 10 \mathrm{mg} / \mathrm{l}$. FINS was detected using the Beckman Access DXI 800 chemiluminescence analyzer. HOMA-IR was defined by the equation: HOMA-IR = [fasting blood glucose (mmol/l) x FINS (mU/l)]/22.5. The normal range of FINS was 3.0-24.9 U/ml, while that of HOMA-IR was $1 \mathrm{U} / \mathrm{ml}$.

The detection of the GRACE risk score was based on the variables of the Killp classification: Systolic blood pressure, heart rate, age, creatine kinase values, whether cardiac arrests and ST segment depression occurred before admission and other items. A total score less than 99 points represented patients under low-risk conditions, a score ranging from 100 to 200 points represented patients under medium-risk conditions, and a score higher than 200 points represented patients under high-risk conditions.

Statistical analysis. The SPSS 19.0 software (SPSS, Inc., Chicago, IL, USA) was used in this study. Measurement data were expressed as mean \pm standard deviation (mean $\pm \mathrm{SD}$ ). The comparison of the mean values between the two groups was performed using the t-test. The comparison between the incidence rates of the two groups was performed using the $\chi^{2}$ test. All the patients were followed up for 12 months. The multivariate logistic regression analysis was used for the analysis of the occurrence of cardiovascular events. $\mathrm{P}<0.05$ was considered to indicate a statistically significant difference.

\section{Results}

Comparison of changes in blood glucose between patients with cardiovascular events and those without cardiovascular events. The average fasting blood glucose and $2 \mathrm{~h}$ postprandial blood glucose levels in the non-cardiovascular event group were lower than those in the cardiovascular events group, $(\mathrm{P}<0.05)$ (Table I).

Comparisons of FINS and HOMA-IR between patients with cardiovascular and those without cardiovascular events. In the non-cardiovascular event group, the average FINS level was $4.26 \pm 1.20 \mathrm{mU} / 1$ and the HOMA-IR level was $0.95 \pm 0.17 \mathrm{mU} / 1$, both were significantly higher than those in 


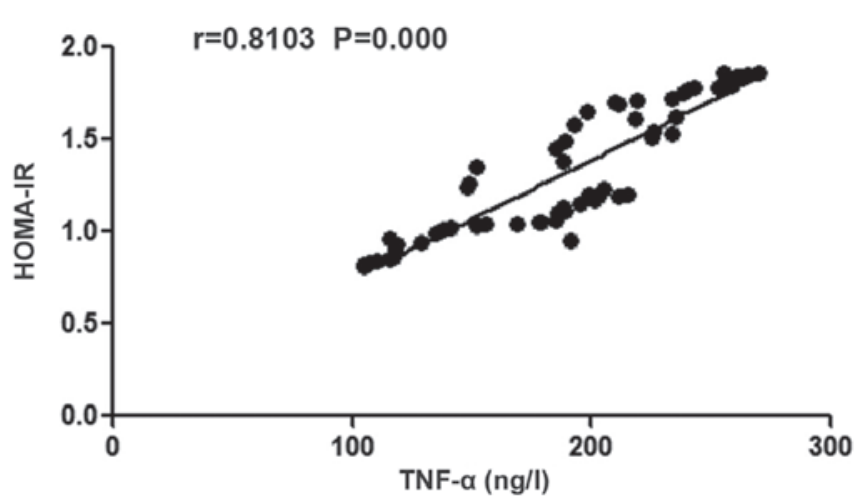

Figure 2. Analysis of the correlation between TNF- $\alpha$ and HOMA-IR. There is a positive correlation between body's TNF- $\alpha$ and HOMA-IR in patients with T2DM complicated with CHD. TNF- $\alpha$, tumor necrosis factor- $\alpha$; HOMA-IR, homeostasis model assessment of insulin resistance indexes; T2DM, diabetes mellitus type 2; CHD, coronary heart disease.

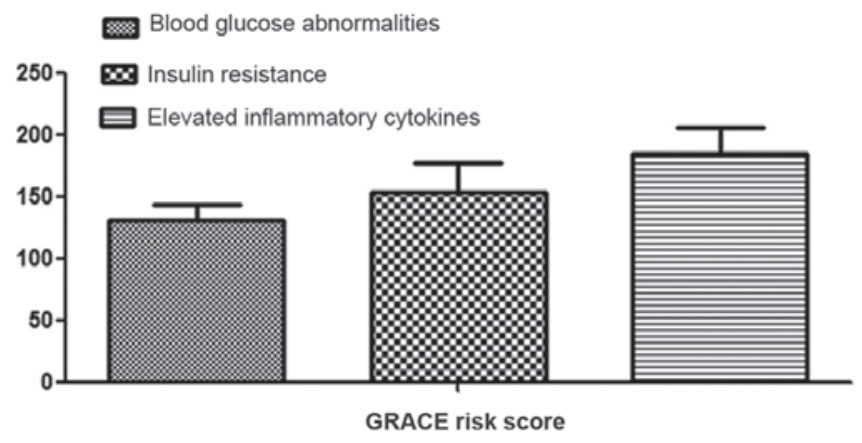

Figure 3. Comparisons of GRACE risk score among patients with blood glucose abnormalities, insulin resistance and elevated inflammatory cytokines. GRACE, Global Registry of Acute Coronary Events.

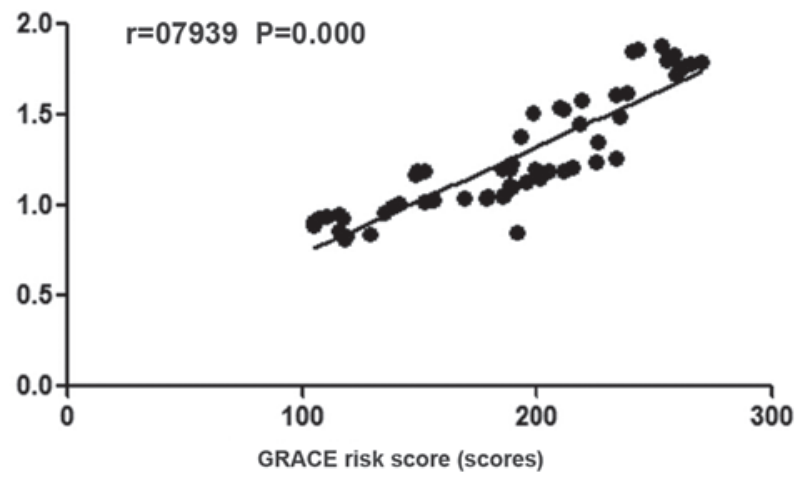

Figure 4. Analysis of the correlation between insulin resistance and GRACE risk score. There is a positive correlation between HOMA-IR and GRACE risk score in patients with T2DM complicated with CHD. GRACE, Global Registry of Acute Coronary Events; HOMA-IR, homeostasis model assessment of insulin resistance indexes; T2DM, diabetes mellitus type 2; CHD, coronary heart disease.

the cardiovascular event group $(\mathrm{P}<0.05)$ (Fig. 1). The levels of FINS and HOMA-IR in the non-cardiovascular event group were significantly higher than those in the cardiovascular event group $(\mathrm{P}<0.05)$.
Table II. Comparison of the inflammatory cytokine levels between patients with cardiovascular events and those without cardiovascular events (mean $\pm \mathrm{SD}$ ).

\begin{tabular}{lccr}
\hline Groups & TNF- $\alpha(\mathrm{ng} / \mathrm{l})$ & $\mathrm{IL}-6(\mathrm{ng} / \mathrm{l})$ & $\mathrm{CRP}(\mathrm{mg} / \mathrm{l})$ \\
\hline Non-cardiovascular & $105.3 \pm 5.6$ & $79.6 \pm 3.4$ & $6.3 \pm 0.1$ \\
Cardiovascular & $258.1 \pm 11.5$ & $125.3 \pm 5.1$ & $11.3 \pm 1.6$ \\
t-test & 75.552 & 47.155 & 19.726 \\
P-value & $<0.05$ & $<0.05$ & $<0.05$ \\
\hline
\end{tabular}

SD, standard deviation, TNF- $\alpha$, tumor necrosis factor- $\alpha$; IL- 6 , interleukin-6; CRP, C-reactive protein.

Comparison of the inflammatory cytokine levels between patients with cardiovascular events and those without cardiovascular events. The levels of TNF- $\alpha$, IL- 6 and CRP in the non-cardiovascular events group were significantly lower than those in the cardiovascular event group $(\mathrm{P}<0.05)$ (Table II).

Analysis of the correlation between TNF- $\alpha$ and HOMA-IR. There was a positive correlation between the TNF- $\alpha$ blood level and the body's HOMA-IR in patients with T2DM complicated with $\mathrm{CHD}(\mathrm{r}=0.8103, \mathrm{P}<0.05)$ (Fig. 2).

Comparisons of GRACE risk score among patients with blood glucose abnormalities, insulin resistance and elevated inflammatory cytokines, and analysis of the correlation between insulin resistance and GRACE risk score. The GRACE risk score of patients with blood glucose abnormalities was $131.2 \pm 12.7$, that of patients with insulin resistance was $153.5 \pm 24.5$, and that of patients with elevated inflammatory cytokines was $185.3 \pm 21.3$; which showed that GRACE risk scores in patients with elevated inflammatory cytokines were higher than those of other patients $(\mathrm{P}<0.05)$. There was a positive correlation between HOMA-IR and GRACE risk score in patients with T2DM complicated with CHD ( $\mathrm{r}=0.7939, \mathrm{P}=$ not significant) (Figs. 3 and 4). GRACE risk scores in patients with elevated inflammatory cytokines are higher than those in patients with insulin resistance and those in patients with blood glucose abnormalities $(\mathrm{P}<0.05)$.

Comparison of the incidence rate of cardiovascular events in the included patients. The incidence rate of cardiovascular events in patients with blood glucose abnormalities was lower than that in patients with insulin resistance and that in patients with elevated inflammatory cytokines $(\mathrm{P}<0.05)$ (Fig. 3 and Table III).

Logistic regression analysis of the incidence of cardiovascular events in the included patients. The occurrence of cardiovascular events was regarded as a dependent variable, and blood glucose abnormalities, insulin resistance and elevated inflammatory cytokines were taken as independent variables. Logistic regression analysis was conducted and the results showed that blood glucose abnormalities, insulin resistance and elevated inflammatory cytokines were all 
Table III. Comparison of the incidence rate of cardiovascular events in the included patients (n, \%).

\begin{tabular}{|c|c|c|c|c|c|c|c|}
\hline Events & Death & $\begin{array}{l}\text { Heart } \\
\text { failure }\end{array}$ & $\begin{array}{l}\text { Myocardial } \\
\text { infarction }\end{array}$ & $\begin{array}{l}\text { Recurrent myocardial } \\
\text { infarction }\end{array}$ & $\begin{array}{l}\text { Angina } \\
\text { pectoris }\end{array}$ & $\begin{array}{l}\text { Recurrent } \\
\text { angina }\end{array}$ & $\begin{array}{l}\text { Total incidence } \\
\text { rate }\end{array}$ \\
\hline $\begin{array}{l}\text { Blood glucose } \\
\text { abnormalities }\end{array}$ & 1 & 1 & 1 & 1 & 2 & 3 & $22.5 \%$ \\
\hline Insulin resistance & 2 & 3 & 4 & 5 & 2 & 4 & $50.0 \%$ \\
\hline $\begin{array}{l}\text { Elevated } \\
\text { inflammatory cytokines }\end{array}$ & 3 & 4 & 5 & 6 & 6 & 5 & $72.5 \%$ \\
\hline$\chi^{2}$ & - & 21.064 & & & & & \\
\hline $\mathrm{P}$-value & - & $<0.05$ & & & & & \\
\hline
\end{tabular}

Table IV. Logistic regression analysis results of the incidence of cardiovascular events in the included patients.

\begin{tabular}{lcccc}
\hline Events & $\mathrm{B}$ & $\mathrm{SE}$ & $\mathrm{OR}$ & $\mathrm{P}$-value \\
\hline $\begin{array}{l}\text { Blood glucose } \\
\text { abnormalities }\end{array}$ & 0.782 & 0.033 & 2.034 & 0.031 \\
$\begin{array}{l}\text { Insulin resistance } \\
\begin{array}{l}\text { Elevated inflammatory } \\
\text { cytokines }\end{array}\end{array}$ & 0.684 & 0.021 & 1.827 & 0.021 \\
& & 0.030 & 1.998 & 0.035 \\
\hline
\end{tabular}

independent risk factors for the occurrence of cardiovascular events (Table IV).

\section{Discussion}

The main complications of T2DM are often associated with cardiovascular and cerebrovascular diseases. Studies have shown that more than $75 \%$ of patients with T2DM die of cardiovascular-related diseases (6); that is, two times more than the number in non-diabetic patients (7). For patients with diabetes mellitus, there is a correlation between the risk of CHD and diabetes-related risk factors such as hyperglycemia, hyperlipidemia, insulin resistance, hyperinsulinemia and other factors (8), which leads to atherosclerosis and further aggravates the development of the disease (9). Previous studies have confirmed that the incidence of cardiovascular events in diabetic patients complicated with CHD is related to factors in the patients such as age, obesity, high blood lipids profile, increased blood pressure and other items (10). However, there is still little known about the association of blood glucose abnormalities, insulin resistance or inflammatory responses of the body with T2DM and CHD.

In the present study, we investigated abnormalities in blood glucose, FINS, HOMA-IR and inflammatory cytokines in patients with or without cardiovascular events. It was found that the fasting blood glucose and the $2 \mathrm{~h}$ postprandial blood glucose levels in the non-cardiovascular events group were lower than those in the cardiovascular event group. However, the levels of FINS and HOMA-IR in the non-cardiovascular event group were significantly higher than those in the cardiovascular event group. The levels of inflammatory factors tumor TNF- $\alpha$, IL-6 and CRP in the non-cardiovascular event group were significantly lower than those in the cardiovascular event group, indicating that the blood glucose abnormalities were relatively more obvious in patients with T2DM complicated with CHD, and there existed relatively more serious insulin resistance with relatively more obvious inflammatory responses.

In addition, we found that GRACE risk scores in patients with elevated inflammatory cytokines were higher than those in patients with insulin resistance and those in patients with blood glucose abnormalities, in agreement with our findings on the incidence rate of cardiovascular events in the same groups of patients. A clear indication that patients with T2DM complicated with CHD are more prone to cardiovascular events. Finally, we conducted the logistic regression analysis and found that abnormal blood glucose abnormalities, insulin resistance and inflammatory cytokines were all independent risk factors for cardiovascular events.

Long-term increased hyperglycemia damages the vascular endothelial cells, promotes the migration and proliferation of vascular smooth muscle cells (11), and activates plasminogen activator inhibitor-1 in the body (12), thus increasing the levels of procoagulant factors as well as antithrombotic factors (13) accelerating the formation of thrombosis and the incidence of cardiovascular events in patients with T2DM complicated with CHD (14). In addition, in patients with T2DM complicated with CHD (15), the body's inflammatory cytokines and their effector cells will lead to cytotoxicity inducing pancreatic $\beta$-cell apoptosis, thereby increasing insulin resistance (16). This will, in turn, induce the adipose tissue cells to secrete more inflammatory factors (17), further aggravating the body's inflammatory response. When insulin resistance manifests itself, the muscle tissues further produce a lot of inflammatory cytokines under the stimulation of high insulin levels in the body (18), which in turn leads to the occurrence of atherosclerosis (19), thus directly leading to the formation of plaques and thrombi in the coronary arteries (20).

The findings in our study support the notion that stronger body inflammatory responses in patients with T2DM complicated with CHD lead to higher incidence rates of cardiovascular events. However, the roles of blood glucose and insulin resistance levels are highlighted by the fact that their levels are also shown to be independent risk factors for cardiovascular events. 


\section{References}

1. Kennedy MW, Fabris E, Suryapranata H and Kedhi E: Is ischemia the only factor predicting cardiovascular outcomes in all diabetes mellitus patients?.Cardiovasc Diabetol 16: 51, 2017.

2. Wallentin L, Lindhagen L, Ärnström E, Husted S, Janzon M, Johnsen SP, Kontny F, Kempf T, Levin LÅ, Lindahl B, et al: FRISC-II study group: Early invasive versus non-invasive treatment in patients with non-ST-elevation acute coronary syndrome (FRISC-II): 15 year follow-up of a prospective, randomised, multicentre study. Lancet 388: 1903-1911, 2016.

3. Völz S, Svedlund S, Andersson B, Li-Ming G and Rundqvist B: Coronary flow reserve in patients with resistant hypertension. Clin Res Cardiol 106: 151-157, 2017.

4. Tripolt NJ, Aberer F, Riedl R, Hutz B, Url J, Dimsity G, Meinitzer A, Stojakovic T, Hödl R, Brodmann M, et al: The effects of linagliptin on endothelial function and global arginine bioavailability ratio in coronary artery disease patients with early diabetes: Study protocol for a randomized controlled trial. Trials 17: 495, 2016.

5. Tsujimoto T, Kajio H and Sugiyama T: Risks for cardiovascular and cardiac deaths in nonobese patients with diabetes and coronary heart disease. Mayo Clin Proc 91: 1545-1554, 2016

6. Jones PH, Bays HE, Chaudhari U, Pordy R, Lorenzato C, Miller K and Robinson JG: Safety of alirocumab (A PCSK9 monoclonal antibody) from 14 randomized trials. Am J Cardiol 118: 1805-1811, 2016

7. Kennedy MW, Fabris E, Ijsselmuiden AJ, Nef H, Reith S, Escaned J, Alfonso F, van Royen N, Wojakowski W, Witkowski A, et al: Combined optical coherence tomography morphologic and fractional flow reserve hemodynamic assessment of non-culprit lesions to better predict adverse event outcomes in diabetes mellitus patients: COMBINE (OCT-FFR) prospective study. Rationale and design. Cardiovasc Diabetol 15: $144,2016$.

8. Konishi H, Miyauchi K, Shitara J, Endo H, Wada H, Doi S, Naito R, Tsuboi S, Ogita M, Dohi T, et al: Impact of lipoprotein(a) on long-term outcomes in patients with diabetes mellitus who underwent percutaneous coronary intervention. Am J Cardiol 118: 1781-1785, 2016

9. Sepah YJ, Sadiq MA, Boyer D, Callanan D, Gallemore R, Bennett M, Marcus D, Halperin L, Hassan M, Campochiaro PA, et al; READ-3 Study group: Twentyfour-month outcomes of the Ranibizumab for edema of the macula in diabetes-Protocol 3 with high dose (READ-3) study. Ophthalmology 123: 2581-2587, 2016

10. Campbell DJ, Tonelli M, Hemmelgarn B, Mitchell C, Tsuyuki R, Ivers N, Campbell T, Pannu R, Verkerke E, Klarenbach S, et al; Interdisciplinary chronic disease collaboration: Assessing outcomes of enhanced chronic disease care through patient education and a value-based formulary study (ACCESS) - study protocol for a $2 \times 2$ factorial randomized trial. Implement Sci 11: 131, 2016.
11. So $\mathrm{CH}$ and Eckman $\mathrm{MH}$ : Combined aspirin and anticoagulant therapy in patients with atrial fibrillation. J Thromb Thrombolysis 43: 7-17, 2017.

12. Sakaguchi Y, Hamano T, Nakano C, Obi Y, Matsui I, Kusunoki Y, Mori D, Oka T, Hashimoto N, Takabatake Y, et al: Association between density of coronary artery calcification and serum magnesium levels among patients with chronic kidney disease. PLoS One 11: e0163673, 2016.

13. Kozyrieva T, Kolesnikova E and Shut I: Correlation of helicobacter pylori infection with development of cardiovascular risk in patients with coronary heart disease in association with type 2 diabetes mellitus. Georgian Med News 9: 24-29, 2016.

14. Gosmanova EO, Mikkelsen MK, Molnar MZ, Lu JL, Yessayan LT, Kalantar-Zadeh K and Kovesdy CP: Association of systolic blood pressure variability with mortality, coronary heart disease, stroke, and renal disease. J Am Coll Cardiol 68: 1375-1386, 2016.

15. Rahmani S, Nakanishi R and Budoff MJ: Imaging atherosclerosis in diabetes: Current state. Curr Diab Rep 16: 105, 2016.

16. Halimi JM, Joly D, Combe C, Choukroun G, Dussol B, Fauvel JP Quéré S and Fiquet B: Blood pressure and proteinuria control remains a challenge in patients with type 2 diabetes mellitus and chronic kidney disease: Experience from the prospective observational ALICE-PROTECT study. BMC Nephrol 17: 135, 2016.

17. Lee SY, Hong MK, Shin DH, Kim JS, Kim BK, Ko YG, Choi D, Jang Y, Kim HS, Valgimigli M, et al: Clinical outcomes of dual antiplatelet therapy after implantation of drug-eluting stents in patients with different cardiovascular risk factors. Clin Res Cardiol 106: 165-173, 2017.

18. Blaha MJ: Personalizing treatment: Between primary and secondary prevention. Am J Cardiol 118: 4-12, 2016.

19. Collins R, Reith C, Emberson J, Armitage J, Baigent C, Blackwell L, Blumenthal R, Danesh J, Smith GD, DeMets D, et al: Interpretation of the evidence for the efficacy and safety of statin therapy. Lancet 388: 2532-2561, 2016.

20. YamamotoE,SugiyamaS,Hirata Y,Tokitsu T,Tabata N,Fujisue K, Sugamura K, Sakamoto K, Tsujita K, Matsumura T, et al: Prognostic significance of circulating leukocyte subtype counts in patients with coronary artery disease. Atherosclerosis 255 : 210-216, 2016. International (CC BY-NC-ND 4.0) License. 